\title{
Asociación entre violencia, estrés y rendimiento académico en alumnos de medicina del primer y sexto años de una universidad pública, 2017
}

\author{
Association between violence, stress and academy performance in $1^{\circ}$ and $6^{\circ}$ year of \\ medical students of a public university, 2017
}

\author{
Lorenzo Barahona-Meza ${ }^{1, a}$, Isabel Amemiya-Hoshi ${ }^{1}$, Elard Sánchez-Tejada ${ }^{1}$, \\ Miguel Oliveros-Donohue ${ }^{1}$, Miguel Pinto-Salinas ${ }^{2}$, Rodney Cuadros-Tairo ${ }^{2}$ \\ ${ }^{1}$ Profesor Principal, Facultad de Medicina, Universidad Nacional Mayor de San Marcos. Lima, Perú. \\ ${ }^{2}$ Estudiante de medicina, Facultad de Medicina, Universidad Nacional Mayor de San Marcos. Lima, Perú. \\ ${ }^{a}$ Doctor en medicina
}

An Fac med. 2018; 79(4):307-11. / DOI: http://dx.doi.org/10.15381/anales.v79i4.15635

\begin{abstract}
Correspondencia:
Lorenzo Barahona Meza

987707995

lorenzo.barahona@yahoo.com.pe

Recibido: 24 de octubre 2018

Aprobado: 14 de noviembre 2018

Conflictos de interés: Los autores

declaran no tener conflictos de interés

Fuente de financiamiento:

Autofinanciado

Citar como: Barahona-Meza L, Amemiya-Hoshi I, Sánchez-Tejada E, Oliveros-Donohue M, Pinto-Salinas M, Cuadros-Tairo R. Asociación entre violencia, estrés y rendimiento académico en alumnos de medicina del primer y sexto años de una universidad pública, 2017. An Fac med. 2018;79(4):307-11. DOI: http://dx.doi.org/10.15381/anales. v79i4.15635
\end{abstract}

\begin{abstract}
Resumen
Introducción. Existen reportes nacionales e internacionales de presencia de estrés en estudiantes de medicina, mayor que en otras carreras profesionales; asimismo, la presencia de estrés se encuentra relacionada a bajo rendimiento académico. Objetivo. Determinar si existe asociación entre violencia, estrés y el rendimiento académico de los alumnos de primer y sexto año de medicina de la Universidad Nacional Mayor de San Marcos. Métodos. Se encuestó a 109 (47\%) alumnos del 6to año, y 122 $(53 \%)$ alumnos del 1er año. La percepción de violencia y estrés se determinaron con instrumentos validados; el rendimiento académico se determinó mediante el promedio ponderado anual. Se utilizó la prueba $U$ de Mann Whitney para establecer diferencias entre las medias de las variables cuantitativas. Para establecer la correlación entre las variables de estudio se utilizó el coeficiente de correlación de Spearman. Valores $p<0,05$ se consideraron como significativos. Resultados. La percepción de violencia fue mayor en estudiantes de 6 to año. Se estableció correlación positiva entre el estrés y violencia en estudiantes de 6 to año. Conclusión. En ambos grupos estudiados se determinó percepción de violencia, mayor en estudiantes de 6to año. La correlación entre violencia y estrés fue significativa sólo en los estudiantes de 6to año. No hubo asociación significativa del estrés y violencia con el rendimiento académico
\end{abstract}

Palabras clave: Violencia; Estrés; Rendimiento Académico; Estudiantes de Medicina

\begin{abstract}
Introduction. There are national and international reports of the presence of stress in medical students, higher than in other professional careers; likewise, the presence of stress is related to low academic performance. Objective. To determine if there is an association between violence, stress and the academic performance of the first and sixth year medical students of the National University of San Marcos. Methods. It was surveyed $109(47 \%)$ students of the 6th year, and $122(53 \%)$ students of the 1 st year. The perception of violence and stress were determined with validated instruments; academic performance was determined by the annual weighted average. The Mann Whitney $U$ test was used to establish differences between the means of the quantitative variables. To establish the correlation between the study variables, the Spearman correlation coefficient was used. Values $p<0,05$ were considered significant. Results. The perception of violence was higher in 6 th grade students. Positive correlation was established between stress and violence in 6th grade students. Conclusion. In both groups studied, perception of violence was determined, higher in 6th grade students. The correlation between violence and stress was significant only in 6th grade students. There was no significant association of stress and violence with academic performance.
\end{abstract}

Keywords: Violence, Stress; Academic Performance; Medical Students 


\section{INTRODUCCIÓN}

El estrés en el área de la salud se puede definir como un fenómeno psicobiológico complejo, de alarma y adaptación que permite al organismo enfrentar situaciones de peligro. Selye en 1954 estableció tres fases en el síndrome de adaptación al estrés: fase de activación general, fase de resistencia y fase de agotamiento ${ }^{1}$.

El estrés produce una respuesta inespecífica y automática, poniendo en alerta todas nuestras capacidades. Puede aparecer ante acontecimientos agradables o en situaciones desagradables de conflicto. La OMS define el estrés como el conjunto de reacciones fisiológicas que prepara al organismo para la acción². Diversos estudios han demostrado que una fuente importante de estrés para los estudiantes es la presión originada por la sobrecarga y evaluación académica de los cursos, autoritarismo docente e incorporación de responsabilidades nuevas y complejas; a estas fuentes se agregan estímulos personales, familiares, sociales, y violencia que se conocen como estresores ${ }^{3,4,5,6}$.

El estrés se ha vinculado con la ansiedad y la depresión. En diversas experiencias al evaluar el rendimiento académico en universitarios de diferentes carreras profesionales, se ha encontrado una interacción entre la ansiedad y situaciones de estrés ${ }^{5,7}$. Al explorarse los efectos adversos de la ansiedad y depresión sobre los resultados académicos en estudiantes de medicina, se encontró que los que tenían niveles elevados de estos estresores, al ser evaluados, presentaron bajo rendimiento académico ${ }^{8,9}$.

La presencia de estrés se ha reportado en el campo de la medicina con mayor frecuencia que en otras carreras profesionales ${ }^{5,8}$, tanto en estudiantes de medicina, como en residentes y médicos jóvenes. Algunos investigadores han considerado al estrés como reto motivador que impulsa el acopio de conocimientos y el trabajo en investigación; lamentablemente, en otros puede ocasionar ansiedad, depresión y deficiencia en la calidad del cuidado de los pacientes ${ }^{9,10}$.

En un estudio previo documentamos la existencia de violencia, entre ellas el bullying, en nuestra institución ${ }^{11}$. Asimismo, el estrés puede incrementarse cuando se asocia a la violencia, asociación que ha sido encontrada en alumnos de postgrado que efectúan investigaciones y en médicos jóvenes ${ }^{12}$. De igual manera, existen reportes sobre la elevada frecuencia de estrés en estudiantes de medicina ${ }^{4,5,6}$. Así, el objetivo de la presente investigación fue determinar la asociación entre el estrés y rendimiento académico en los estudiantes de medicina de primer y sexto año de estudios en la Univerisidad Nacional Mayor de San Marcos.

\section{MÉTOdOS}

Se realizó una investigación observacional, descriptiva y transversal, para evaluar la asociación de estrés con violencia y el rendimiento académico. Se aplicó el método encuesta y la técnica del cuestionario autoaplicado. La muestra estuvo conformada por 231 estudiantes del 1er y 6 to año de estudios de medicina de la Universidad Nacional Mayor de San Marcos (UNMSM), con matrícula regular. Las encuestas se aplicaron en el año 2017.

La variable violencia se evaluó como percepción por parte del estudiante, con los siguientes 12 ítems del instrumento validado por la Defensoría del Pueblo de España, corregido y validado por Hoyos y col. ${ }^{11}$ : ignorar, no dejar participar, insultar, poner apodos, hablar mal de él o de ella, esconder cosas, romper cosas, pegar, amenazar con intimidar, acosar sexualmente, obligar a hacer cosas que no quiere, amenazar con arma blanca o de fuego.

El variable estrés se evaluó como percepción con la escala unidimensional para estrés estudiantil de Barraza ${ }^{13}$, que consta de 15 ítems con respuestas posibles tipo Likert (nunca, algunas veces, casi siempre y siempre), y consta de los siguientes enunciados: el tener que asistir a clases me cansa, los problemas relacionados con mis estudios me deprimen fácilmente, durante las clases me siento somnoliento, creo que estudiar hace que me sienta agotado, cada vez me es más difícil concentrarme en las clases, me desilusionan mis estudios, antes de terminar las clases ya me siento cansado, no me interesa asistir a clases, cada vez me cuesta más trabajo ponerle atención al maestro, el asistir a clases se me hace aburrido, siento que estudiar me está desgastando físicamente, cada vez me siento más frustrado por ir a la escuela, no creo terminar con éxito mis estudios, siento que tengo más problemas para recordar lo que estudio, creo que estudiar me está desgastando emocionalmente.

El rendimiento académico se estudió utilizando el indicador cuantitativo de la nota al final del segundo semestre académico, que fue obtenida como promedio ponderado de los cursos matriculados por los estudiantes.

Se utilizó estadística descriptiva para la presentación de los datos; asimismo, se utilizó la prueba U de Mann Whitney para establecer diferencias entre las medias de las variables cuantitativas y Chi cuadrado para la variable cualitativa sexo. Para establecer la correlación entre las variables de estudio se utilizó el coeficiente de correlación de Spearman. Valores $p<0,05$ se consideraron como significativos.

El presente estudio tuvo la aprobación del Comité de Ética de la Facultad de Medicina de la UNMSM. Los participantes firmaron el respectivo consentimiento informado. Se aseguró la confidencialidad de la información a través del uso de códigos de identificación conocidos solamente por los investigadores.

\section{RESULTADOS}

Se evaluaron 231 encuestas auto aplicadas de alumnos de medicina de 1ero y 6 to año de estudios. La distribución de las encuestas fue: 109 encuestas (47\%) de alumnos del 6to año, y 122 encuestas (53\%) de alumnos del 1er. año. La relación varones/mujeres fue de 1,1. Las variables edad, promedio ponderado, puntaje sobre violencia y puntaje sobre estrés no presentaron distribución normal; las características descriptivas de dichas variables se presentan en la tabla 1.

No existieron diferencias significativas en la variable sexo del estudiante según año de estudio ( $p=0,645)$, ni en el puntaje sobre estrés $(0,536)$. Se encontrararon diferencias significativas según año de es- 
Tabla 1. Edad, promedio ponderado y puntaje de violencia y estres de alumnos de medicina del 1ero y 6to año de estudios de la UNMSM, 2017

\begin{tabular}{|c|c|c|c|c|c|c|c|c|}
\hline & \multicolumn{8}{|c|}{ Año de estudios } \\
\hline & \multicolumn{4}{|c|}{$1^{\circ}$ año $\left(\mathrm{N}^{\circ} 122\right)$} & \multicolumn{4}{|c|}{$6^{\circ}$ año $\left(N^{\circ} 109\right)$} \\
\hline & Min & Max & Media & DS* & Min & Max & Media & DS \\
\hline Edad & 14 & 36 & 19,59 & 2,58 & 21 & 38 & 25,36 & 2,34 \\
\hline Promedio ponderado & 8,429 & 16,412 & 14,07 & 1,346 & 11,864 & 16,609 & 14,862 & 0,762 \\
\hline Puntaje sobre violencia & 0 & 6 & 0,76 & 1,13 & 0 & 7 & 1,22 & 1,48 \\
\hline Puntaje sobre estrés & 8 & 44 & 26,91 & 5,59 & 6 & 48 & 26,66 & 6,19 \\
\hline
\end{tabular}

DS: desviación estándar

tudio, en la edad promedio del estudiante, en el promedio ponderado del rendimiento $y$ en el puntaje sobre violencia $(p=0,000, p=0,000, p=0,015$, respectivamente), tal como se aprecia en la tabla 2.

Se determinó correlación positiva débil y significativa entre las variables estrés y violencia en los estudiantes de medicina de 6 to año, tal como se aprecia en la tabla 3 y figura 1. Las demás correlaciones no fueron significativas, tal como se observa en la tabla 3.

\section{DISCUSIÓN}

La salud mental es un serio problema de salud pública en aumento, y no controlado, uno de sus indicadores es el consumo de alcohol y drogas. Los estudiantes universitarios no escapan a esta realidad, que además puede afectar en alguna medida su desempeño académico y social. Además, la salud mental de los estudiantes que ingresan a una universidad puede afectarse por las exigencias académicas, que plantean situaciones de éxito o fracaso dependiendo del desarrollo de capacidades que se deben adquirir en el curso de los años de formación ${ }^{4}$.

La educación médica capacita y forma médicos competentes y profesionales capaces de atender a los enfermos del país, avanzar en la ciencia de la medicina y promover la salud pública. Para lo cual es necesario que al ingreso se pueda identificar individuos aptos y con el compromiso necesario para seguir una carrera en medicina basada en la comprensión de las demandas, desafíos y recompensas de la profesión ${ }^{14}$. Estos desafíos y demandas se convierten en riesgos que para los estudiantes de medicina están incrementados debido a intensos momentos de presión académica, carga de trabajos y tareas así como problemas financieros y familiares complementarios al ser una población de escasos recursos financieros, poco tiempo para dormir y descansar, contacto directo con el sufrimiento y muerte, abuso de estudiantes, y un "currículum oculto" de cinismo que pueden contribuir en la disminución en la salud mental de los estudiantes ${ }^{6}$.
Los resultados de nuestra investigación muestran que el estrés medido con la escala de Barraza se encuentra presente en los estudiantes de medicina de primero y sexto año, pero en niveles promedio bajos, puesto que el máximo nivel fue de 44 de 60 puntos totales de la escala. Se considera que el entrenamiento médico genera estrés y es inevitable durante los estudios médicos y también durante la práctica médica, como se han reportado en otros estudios que abordan este tema ${ }^{5,8}$, tanto en estudiantes de medicina, como en residentes y médicos jóvenes. A pesar que se ha sugerido que el estrés puede ser un estímulo para mejorar el acopio de conocimientos, esta condición cuando se encuentra relacionada a la angustia, depresión, producen alteración de la salud mental y deficiencia en la

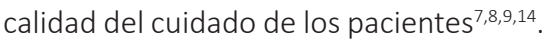

La correlación entre el estrés y rendimiento académico, este último medido a través del promedio ponderado, fue negativa débil y no significativa. Estos resultados no confirman lo encontrado

Tabla 2. Diferencias entre variables sexo, edad, promedio ponderado, violencia y estrés de alumnos de medicina del 1 ero y 6 to año de estudios de la UNMSM, 2017

\begin{tabular}{lccc} 
Variables & \multicolumn{3}{c}{ Año de estudios } \\
\cline { 2 - 4 } & 6to. año & 1er. año & $\mathbf{p}$ \\
Sexo & $57(45,6 \%)$ & $68(54,4 \%)$ & 0,645 \\
$\quad$ Masculino & $52(49,5 \%)$ & $53(50,5 \%)$ & $0,000^{*}$ \\
$\quad$ Femenino & $25,3+/-2,9$ & $19,5+/-2,5$ & $0,000^{*}$ \\
Edad (años) & $14,8+/-0,7$ & $14+/-1,3$ & $0,015 *$ \\
Promedio Ponderado & $1,2+/-1,4$ & $0,7+/-1,1$ & 0,536 \\
Puntaje sobre violenci & $26,6+/-6,2$ & $26,9+/-5,6$ & \\
Puntaje sobre estrés & & &
\end{tabular}

* Estadísticamente significativo, $\mathrm{p}<0,05$ 
Tabla 3. Correlación de Spearman entre promedio ponderado, puntaje sobre violencia y puntaje sobre estrés de alumnos de medicina del 1ero y 6 to año de estudios de la UNMSM, 2017

\begin{tabular}{|c|c|c|c|c|}
\hline Año de estudio & Variable1 & Variable 2 & Correlación de Spearman & $\mathbf{p}$ \\
\hline \multirow{3}{*}{ 1er.año } & Puntaje sobre violencia & Puntaje sobre estrés & 0,154 & 0,090 \\
\hline & Puntaje sobre violencia & Promedio ponderado & $-0,049$ & 0,597 \\
\hline & Puntaje sobre estrés & Promedio ponderado & $-0,005$ & 0,958 \\
\hline \multirow{3}{*}{ 6to.año } & Puntaje sobre violencia & Puntaje sobre estrés & 0,292 & $0,002^{*}$ \\
\hline & Puntaje sobre violencia & Promedio ponderado & $-0,027$ & 0,792 \\
\hline & Puntaje sobre estrés & Promedio ponderado & $-0,041$ & 0,684 \\
\hline
\end{tabular}

* Estadísticamente significativo, $\mathrm{p}<0,05$

por otros investigadores quienes sugirieron que la angustia entre los estudiantes puede influir en su rendimiento académico, ausentismo, concentración y la deshonestidad académica ${ }^{8,9,15}$.

La violencia también estuvo presente en los resultados de nuestra investigación; sin embargo, sus niveles fueron también bajos. De un puntaje máximo de 12 , los estudiantes de sexto año señalaron haber tenido en promedio menos de dos y los de primer año, menos de 1 , diferencia estadísticamente significativa y comparable a otras investigaciones que señalan que la violencia se incrementa en los estudiantes de medicina en los años superiores. Silver determinó que al ingreso a la universidad los estudiantes reportaron un $46,4 \%$ de abuso y se incrementó a $80,6 \%$ en los estudiantes de los últimos años $^{16}$. La violencia también se reportó anteriormente en estudiantes de medicina del sexto año en nuestra universidad ${ }^{11}$ $y$ en otra universidad peruana ${ }^{17}$.

El fenómeno de violencia y abuso en la escuela de medicina es frecuente pero poco estudiado. Según Henry Silver en $1982^{18}, 1984^{19}$, y $1990^{16}$, los estudiantes aprenden no lo que los profesores dicen, sino lo que hacen, y la presencia de si-

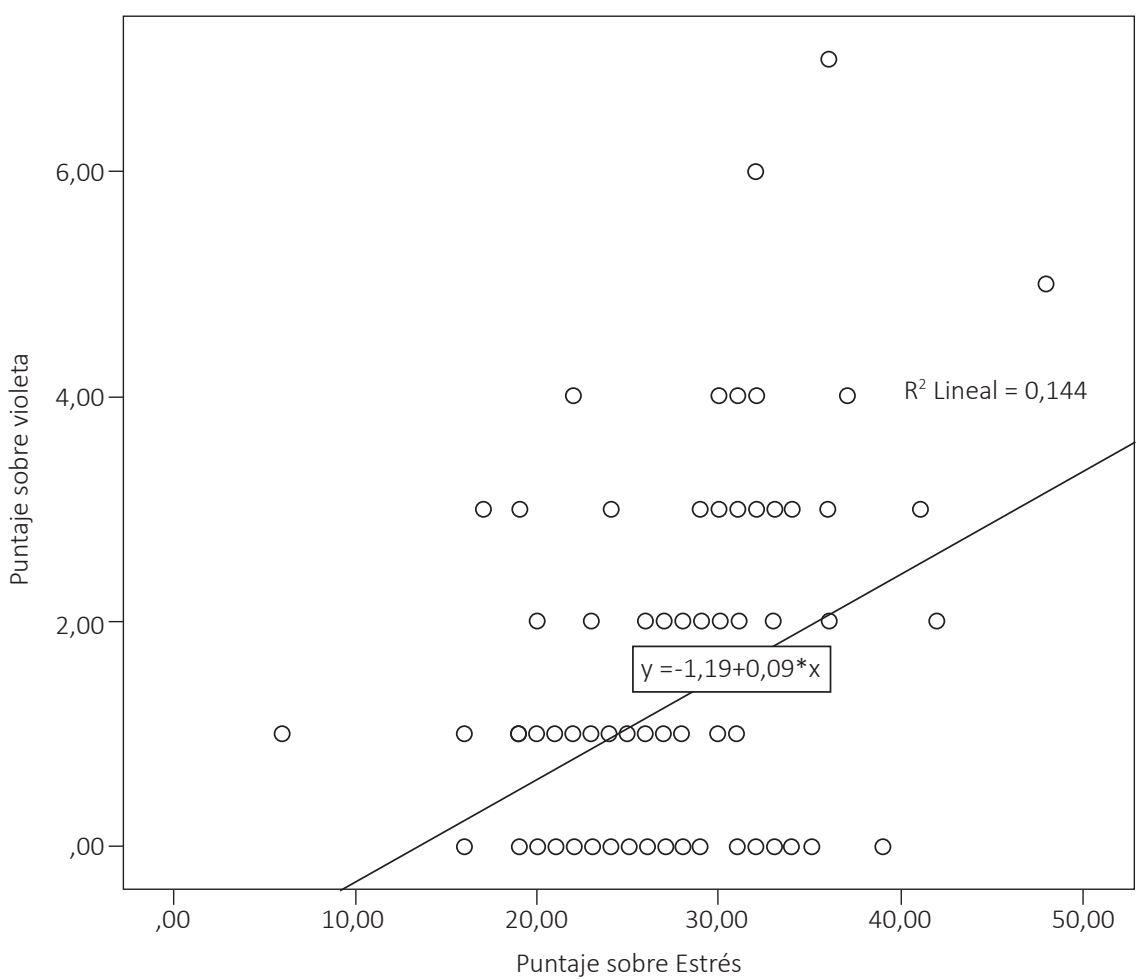

Figura 1. Correlación entre puntaje sobre estrés y puntaje sobre violencia en alumnos de medicina del 6to año de estudios de la UNMSM, 2017 tuaciones abusivas causa en ellos estres significativo. Los resultados de Silver no se confirmaron de manera concluyente en esta investigación, que encontró una correlación positiva débil pero significativa entre estrés y violencia, solamente en estudiantes de sexto año de medicina, sin estar ninguno de los dos correlacionados con el promedio ponderado de la nota final del año en curso. La importancia de estas comparaciones no es discutible, ya que la angustia y el estrés, así como la violencia en los dos grupos, no debe descartarse, al margen de cómo se comparen con el rendimiento académico. Es probable que el rendimiento se encuentre asociado a otras variables cuya influencia sea mayor. Además, se ha determinado en graduados de facultades de medicina, que el estrés puede afectar negativamente la profesionalidad y la atención del paciente ${ }^{14}$.

La limitación de nuestra investigación radica en que es un estudio de percepciones, pues explora una subjetividad que puede producir sesgos en su medición cuantitativa. Además, los resultados no podrán ser extrapolados y serán válidos solo para este grupo poblacional de la facultad de medicina.

En conclusión, según nuestros hallazgos, existe percepción de violencia y estrés en estudiantes de medicina del 1er y 6 to año de estudios de la Facultad de Medicina de la UNMSM. Al compararse los puntajes de violencia entre los alumnos de 1 er y 6 to año, se encontró una diferencia significativa en los alumnos que están finalizando sus estudios. De igual manera, la correlación entre violencia y estrés solo fue significativa en los alumnos de 6to año. No hubo asociación significativa con el rendimiento académico. Esta violencia, 
que se incrementó con la progresión de la carrera, es preocupante y constituye un reto para la universidad el apoyo y seguimiento a los afectados así como el fortalecimiento de la formación ética, la cual debe ser impulsada desde el ingreso de los estudiantes a nuestra facultad.

\section{REFERENCIAS BIBLIOGRÁFICAS}

1. Selye H. Stress and disease. Science. 1955;122(3171):625-31.

2. World Health Organization [Internet]. International Labour Organization and Who Committee on Occupational Health. Psychosocial Factors at Work: Recognition and Control. Occupational Safety and Health Series $N^{\circ} 56,1984$. [Fecha de acceso 23 de mayo 2018]. Disponible en: https://tinyurl. com/ybcsx 5 qf

3. Sociedad Española para el estudio de la Ansiedad y el Estrés [Internet]. La naturales del estrés, Dr. Antonio Cano Vindel. . [Fecha de acceso 23 de mayo 2018]. Disponible en: https://tinyurl.com/ y9voxqer

4. Rodríguez M, Sanmiguel M, Muñoz A, Rodriguez C. El estrés en estudiantes de medicina al inicio y final de su formación académica. Revista Iberoamericana de Educación. 2014;66:105-122

5. Celis J, Bustamante M, Cabrera D, Cabrera M, Alarcon W, Monge E. Ansiedad y Estrés Acadé- mico en Estudiantes de Medicina Humana del Primer y Sexto Año Anales Facultad Medicina. 2001; 62(1):25-30. DOI: http://dx.doi.org/10.15381/ anales.v62i1.4143

6. Castillo C, Chacón T, Diaz-Véliz G. Ansiedad y fuentes de estrés académico en estudiantes de carreras de la salud. Inv Ed Med. 2016; 5(20):230-237. DOI: http://dx.doi.org/10.1016/j. riem.2016.03.001

7. Quine L. Workplace bullying in NHS community trust: staff questionnaire Psurvey. BMJ. 1999;318(7178):228-32

8. Gonzales-Olaya H, Delgado-Rico H, EscobarSánchez M, Cárdenas-Angelone ME. Asociación entre el estrés, el riesgo de depresión y el rendimiento académico en estudiantes de los primeros semestres de un programa colombiano de medicina. FEM. 2014;17(1):47-54. DOI: http:// dx.doi.org/10.4321/S2014-98322014000100008

9. Quine L. Workplace bullying in junior doctors: questionnaire survey. BMJ. 2002; 324(7342):878-9. DOI: https://doi.org/10.1136/bmj.324.7342.878

10. Dahlin M, Joneborg N, Runeson B. Stress and depression among medical students: a cross sectional study. Med Educ 2005;39(6):594-604 DOI:10.1111/j.1365-2929.2005.02176.x

11. Oliveros M, Perales A, Zavala S, Amemiya I, Pinto M, Ramos A. Percepcion de bullying en alumnos de sexto año de una universidad pública de Lima 2015. An Fac med. 2016;77(3):231-6. DOI: http:// dx.doi.org/10.15381/anales.v77i3.12406

12. Stebbing J, Mandalia S, Portsmouth S, Leonard P, Crane J, Bower M, Earl H, Quine L. A querstionnaire surey of stress and bullying in doctors undertaking research. Postgrad Med J. 2004; 80:93-96. DOI: http://dx.doi.org/10.1136/pmj.2003.009001

13. Barraza A. Validación psicométrica de la escala unidimensional del burnout estudiantil. Revista Intercontinental de Psicología y Educación. 2011;13(2):51-74.

14. Dyrbye LN, Thomas MR, Shanafelt TD. Systematic review of depression, anxiety, and other indicators of psychological distress among U.S. and Canadian medical students. Academic Medicine. 2006;81(4):354-73

15. Gutiérrez J, Montoya L, Toro B, Briñón M, Rosas E, Salazar L. Depresión en estudiantes universitarios y su asociación con el estrés académico. Rev CES Med. 2010;24(1):7-17

16. Silver HK, Glicken AD. Medical student abuse. Incidence, severity and significance. JAMA. 1990;263(4): 527-32.

17. Munayco-Guillén F, Cámara-Reyes A, MuñozTafur J, Arroyo-Hernández H, Mejia R, Lem-Arce $F$, et al. Características del maltrato hacia estudiantes de medicina de una universidad pública del Perú. Rev Peru Med Exp Salud Pública. 2016;33(1):58-66. DOI: http://dx.doi.org/10.17843/ rpmesp.2016.331.2008

18. Silver HK. Medical students and medical school. JAMA. 1982; 247(3):309-10.

19. Rosenberg DA, Silver HK. Medical student abuse. An unnecessary and preventable cause of stress. JAMA. 1984; 251(6):739-42. 